Journal of Applied Fluid Mechanics, Vol. 9, Special Issue 1, pp. 31-37, 2016.

Selected papers from the $7^{\text {th }}$ International Exergy, Energy

and Environment Symposium, IEEE7-2015

Available online at www.jafmonline.net, ISSN 1735-3572, EISSN 1735-3645.

DOI: $10.36884 /$ jafm.9.SI1.26394

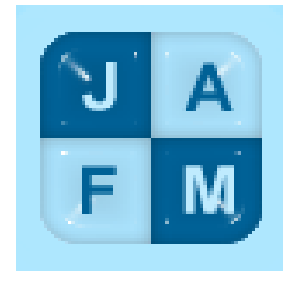

\title{
Aero-Thermal Optimization of a Heat Sink using Variable Neighbourhood Search
}

\author{
P. Jandaud ${ }^{\dagger}$, L. Lambourg and S. Harmand \\ University of Valenciennes, LAMIH, CNRS UMR 8201, Campus le Mont Houy, Valenciennes, F59313, \\ France
}

$\dagger$ Corresponding Author Email: pierre-olivier.jandaud@univ-valenciennes.fr

(Received October 20, 2015; accepted December 10, 2015)

\begin{abstract}
This paper presents a geometrical optimization of a heat sink modelled using three-dimensional CFD. The heat sink studied is circular with radial inlets and parallel fins. The parameters of the optimization are the different spacings between the fins. The optimization process is multi-objective and uses an aggregated objective function of both the thermal resistance and the pressure drop of the system. To perform the optimization, a relatively new technique has been used called Variable Neighbourhood Search (VNS). The optimization results give several interesting new geometries. In addition, the performances of VNS are measured with two criteria: the speed of convergence and the repeatability between two optimization runs. These performances are good compared to more traditional optimization techniques like Genetic Algorithms.
\end{abstract}

Keywords: Heat sinks; Electronics cooling; Multi-ojective; Optimization; Heat transfer; CFD.

\section{NOMENCLATURE}

$\begin{array}{ll}\mathrm{DP} & \text { pressure drop } \\ \mathrm{d} & \begin{array}{l}\text { relative distance between the optimums } \\ \text { pressure coefficient }\end{array} \\ \mathrm{f} & \text { objective function } \\ f_{\text {count }} & \text { number of objective function evaluation } \\ \mathrm{k} & \text { number of neighbourhoods } \\ \mathrm{P} & \text { pressure } \\ \mathrm{Q} & \text { volume flow rate } \\ \mathrm{R} & \text { thermal resistance } \\ \mathrm{S} & \text { surface of the inlet } \\ \mathrm{T} & \text { temperature } \\ \mathrm{u} & \text { velocity } \\ \mathrm{x} & \text { fin spacing } \\ & \\ \text { Greek letters } \\ \alpha & \text { weigth coefficient }\end{array}$

\section{INTRODUCTION}

Heat sinks are widely used for cooling electronic components. On car alternators, a heat sink is placed on the back of the machine to cool the rectifier bridge and it also allows air to enter the machine to cool the windings of the stator and the rotor. As a result, a well-designed heat sink should dissipate the heat produced by the electronic components but also should not affect the air flow.

\author{
$\lambda \quad$ thermal conductivity \\ $\rho \quad$ density \\ $\Phi \quad$ heating power \\ Superscipts \\ * optimal \\ Subscripts \\ $0 \quad$ at the inlet \\ base mean value at the base \\ max maximum value \\ mean mean value \\ min minimum value \\ Ref reference value
}

Its design is a multi-objective optimization problem with two objectives to minimize: the pressure drop of the system and its thermal resistance.

Many studies can be found on the optimization of heat sinks, some recent examples are found in Karathanassiset al. (2013) and Lin et al. (2014). However, most of them use simple model for the heat sink and/or local optimization algorithms. In the first study (Karathanassis, 2013), the heat sink is 
modelled using an equivalent thermal resistance network. The geometry is simple and flow rates distribution inside the heat sink has to be known to obtain the different convective heat transfer coefficient needed. The optimization method used is a genetic algorithm which needs around $10^{4}$ evaluation of the objective to reach an optimum for three design parameters. In the second study (Lin 2014), a three-dimensional CFD model is used for optimization. Only one channel of the heat sink is modelled.The flow is laminar and the grid used has approximately 150000 elements. Optimization is performed using a conjugate-gradient method which gives only local optimums but three different starting points were tested.

In industrial applications, the geometries of the heat sinks can be complex: the fins are not necessarily parallel or straight, the flow distribution is not necessarily uniform... To numerically study these heat sinks, complex three-dimensional CFD models are needed. Each time an evaluation of the objective is needed in the algorithm, a CFD run has to bedone.Therefore, optimization becomes more time-consuming and the optimization method should be chosen carefully.

In the field of optimization, two main families of method exist. The first kind of algorithms are based on gradient methods and suited for local optimization of continuous function. The second kind isstochastic algorithms based on the evolution of a population like Genetic Algorithms and Particle Swarm Optimization(Kennedy et al. 1995). These methods are the most popular in heat transfer engineering (Gosselinet al, 2008) due to their global aspect and their simplicity in implementation.However, they can require extensive numerical resources. Some examples of their use in thermal problems can be found in Bornschlegellet al. (2012) and Jandaudet al. (2012). In these two studies, the optimization is performed on simple lumped method based model.Three studies by Smolka (2013), Kim et al (2007) and Vasudevet al (2014) shares similarities. They couplegenetic algorithms and a 3D CFD model and use around five optimization parameters. In these studies, due to the numerical cost of the computations, the optimization process is stopped after a set number of generations and the results are assumed to be the global optima. The main problem is the user of the method has to know a priori the right number of generations and the size of the population for his case. An underestimated value of these parameters can give a non-optimal result and an overestimated value will result in wasted numerical resources.

In this paper, the optimization process is performed using a metaheuristic called Variable Neighbourhood Search (VNS) created by Mladenovicet al. (1997). This method is a hybrid between gradient based methods and stochastic metaheuristics. It creates a series of neighbourhoods of growing sizes around a potential optimum. In each of these neighbourhoods, a local search is done. If a better optimum is found, a new set of neighbourhoods is created and the previous steps are repeated until a stopping criterion is met. This method is less time consuming than population based algorithm: at each generation it focuses only on one point instead of a whole population. This method is more and more used in a wide variety of fields (Mladenovicet al. 2010). The variant of VNS used in this paper has been proposed by Libertiet al. (2005) and is fitted for global continuous optimization of constrained non-linear problems.

The aim of this article is to set a methodology to use global optimization with a 3D CFD model with relatively low computational time. The method chosen is VNS and it is tested on a simple version of a car alternator heat sink modelled using Fluent ${ }^{\circledR}$. The optimization parameters are the spacing of the fins and the two objectives are to reduce the thermal resistance and/or the pressure drop of the heat sink.

\section{CFD MODEL}

\subsection{Model Description}

The geometry studied is a simpler version of the heat sinks used in car alternators. The base is circular with an open gap in the middle. Twelve heat sources are placed directly on the bottom of the base under the fins. Four groups of eleven parallels fins are disposed on the base. The fins and the base are in aluminum $\left(\lambda=120 \mathrm{~W} \cdot \mathrm{m}^{-1} \cdot \mathrm{K}^{-1}\right)$. A plastic hood covers the heat sink and forces the air flow to enter radially in front of the fins and to exit axially in the centre.Due to symmetries, only one eighth of the geometry is modelled (Fig. 1). The numerical domain reproduces our test bench for the heat sinks of alternators (Figs 1 and 2). The heat sink and its plastic hood are placed on a circular pipe. The air flow is created with an exhaust fan placed at the outlet of the pipe. The maximum total volume flow rate is $12 \mathrm{~L} / \mathrm{s}$ which correspond to a Reynolds number of 21600 .

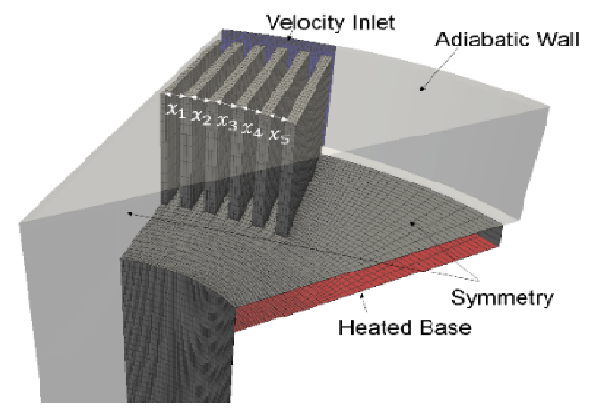

Fig. 1. General view of the domain.

The model uses Reynolds Averaged Navier-Stokes (RANS) equations coupled with the Energy Equation to solve the problem. The turbulence model chosen is k- $\omega$ SST which is well suited for low-Reynolds turbulent flow. The equations are solve with Fluent ${ }^{\circledR}$ using second orderdiscretization schemes. The mesh used is fully hexahedral with approximately 350000 elements. 


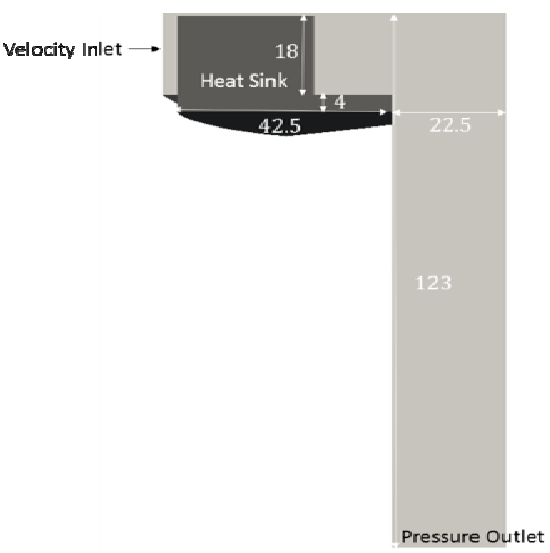

Fig. 2. Dimensions (in $\mathrm{mm}$ ) of the domain.

The surface mesh on the heat sink can be seen on fig 1. At maximum velocity, the average value of $y^{+}$on the heat sink is 1.5 .

\subsection{Boundary Conditions}

At the inlet, the flow enters the heat sink with a temperature of $300 \mathrm{~K}$ and a constant velocity:

$u_{0}=Q / S$

At the outlet, the pressure is kept equal to atmospheric pressure. The sides of the domain are symmetries. The walls corresponding to the plastic hood are considered adiabatic. At the base, to represent the heating of electronic components, the heat sources are circular with a diameter of $13 \mathrm{~mm}$ with a heating power of $11 \mathrm{~W}$ per component (Fig .3).

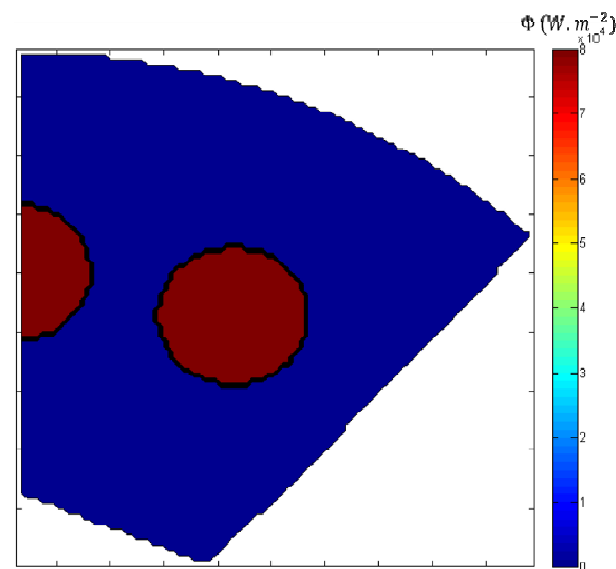

Fig. 3. Heat Flux repartition at the base of the heat sink.

\subsection{Mesh Parameterization}

In order to be able to perform optimization on this model, the mesh has to be parameterized. Our parameters are the different spacings of the fins $x_{i}$ (fig 1). To improve the flow distribution in the channels between the fins, the size of the inlet changes also according to the spacings of fins. The edge of the inlet is aligned with the last fin. As a result, the velocity at the inlet changes with the fins spacings. The different spacings can vary from 0.5 $\mathrm{mm}$ to $3.5 \mathrm{~mm}$. The grid generation, the solver and the post-processing are controlled by Matlab ${ }^{\circledR}$ scripts to be able to use optimization.

\subsection{Experimental Validation}

To validate the results of our numerical a reference heat sink has been tested has been tested experimentally. The reference has a constant spacing of $2 \mathrm{~mm}$ between each fin. The heat flux is controlled at the base with circular electric resistances. The temperature at each resistance is measured with a type $\mathrm{K}$ thermocouple. The power of the fan is variable and allows the flow rate to vary from 2 to $12 \mathrm{~L} / \mathrm{s}$. The comparison is between the experimental and numerical thermal resistance. It is calculated by dividing the temperature by the heating power:

$$
R=\frac{T_{\text {base }}-T_{0}}{\Phi}
$$

The results (Fig. 4) show a good agreement between experimental and numerical results. For the volume flow rates over $4 \mathrm{~L} / \mathrm{s}$, the relative difference does not exceed 5\%. For the lowest flow rate, the relative difference increases to $16 \%$. This difference can be explained by the fact that at low speed the flow is less turbulent and more in a transitional state between laminar and turbulent. Therefore, the turbulent model k- $\omega$ SST may not be valid anymore For the optimization study, the flow is kept constant at $10 \mathrm{~L} / \mathrm{s}$ in the zone showing good agreement bet experimental and numerical results.

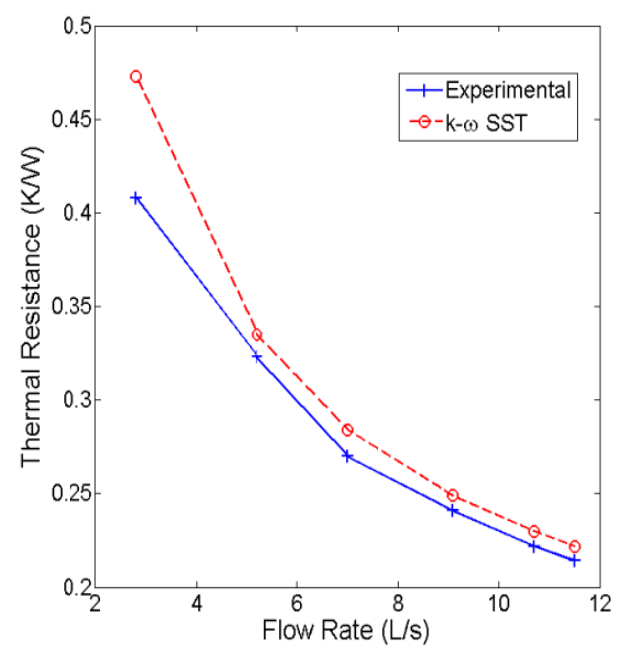

Fig .4. Experimental and numerical results for the Thermal Resistance.

\section{OPTIMIZATION METHODOLOGY}

\subsection{Variable Neighbourhood Search}

Variable NeighbourhoodSearch (VNS) is a hybrid optimization method combining local gradient-based search and stochastic 
elementscreated by Mladenovicet al. (1997). From a random starting point in the optimization space, a local search is done to find a first local minimum. Around this minimum, a number $k$ of neighbourhoods of growing sizes is built. In each neighbourhood, a local search is performed. If a new local minimum is found, a new set of neighbourhoods is built and the local searches restart. The process ends when all the neighbourhoods have been explored.

The version of VNS used comes from Libertiet al. (2005) which allows constrained and non-linear optimization. The local search algorithm used is Sequential Quadratic Programming (SQP) which is directly implemented in Matlab ${ }^{\circledR}$. The neighbourhoods are hyper-rectangles centred on the local minimum. The detailed algorithm of the method can be found below:

\begin{tabular}{l}
\hline VNS Algorithm \\
\hline $\begin{array}{l}\text { Input: Maximum number of Neighbourhoods } k \\
\text { Objective Function } f\end{array}$ \\
\hline $\begin{array}{l}\text { Initialization: } i \leftarrow 1 \text {, pick a random point } x \text { and } \\
\text { do a local search to find a local minimum } x^{*} \text {. }\end{array}$ \\
While $i \leq k$, \\
Create a Neighbourhood $N_{i}\left(x^{*}\right)$ such \\
that $N_{i-1}\left(x^{*}\right) \subset N_{i}\left(x^{*}\right)$ \\
Take a random $x \in N_{i}\left(x^{*}\right)$ \\
Do a local search from $x$ to find a local \\
minimum $x^{\prime}$ \\
Iff $\left(x^{\prime}\right)<f\left(x^{*}\right)$ then $x^{*} \leftarrow x^{\prime}$ and $i \leftarrow 1$ \\
elsei $\leftarrow i+1$ \\
\hline Return $x^{*}$ \\
\hline
\end{tabular}

\subsection{Objective function}

The optimization of the heat sink needs a multi-objectives approach. Both the thermal resistance and the pressure drop have to be taken into accountto be minimized. As a result, an Aggregated Objective Function (AOF) is built which is the weighted average of the thermal resistance and the pressure drop divided by the values of the reference geometry used for experimental validation.

$$
f=\alpha \frac{R}{R_{r e f}}+(1-\alpha) \frac{D P}{D P_{r e f}}
$$

During the optimization several values of the weight coefficient $\alpha$ are used. For each $\alpha$, ten run will be tested with a different random starting point to test the performances and repeatability of the method.The best of the results is considered to be the global optimum.

\subsection{Evaluation of the method}

To evaluate the performances of two criteria are used. The first one is the number of evaluation of the objective function: $f_{\text {count }}$. Each time the function needs to be evaluated a CFD computation has to be done. Therefore, the number of evaluation is a good measurement of time spent for the optimization. The second criterion is, for a fixed value of $\alpha$, the relative difference between the optimums and the best minimum:

$d_{\alpha}\left(x^{*}\right)=\frac{f\left(x^{*}\right)-f_{\min }}{f_{\min }}$

Low values of $d_{\alpha}$ will ensure a good repeatability for the optimization method.

\section{RESULTS}

\subsection{Optimal Geometries}

Optimization has been performed for seven values of $\alpha$ going from 0 (only the pressure drop is minimized) to 1 (only the thermal resistance is minimized). The spacing of the fins can vary from $0.5 \mathrm{~mm}$ to $3.5 \mathrm{~mm}$. All the results are compared to the reference geometry with constant $2 \mathrm{~mm}$ spacing which was used for the experimental validation (Fig 6.a).The different optimal geometry (Table 1) are represented in a Pareto front (Fig. 5). For a multi-objective optimization, the Pareto front shows the boundary between feasible and infeasible solutions.

The first interesting solutions are the extreme values where only one objective is minimized. For the case where thermal resistance is minimized $(\alpha=1)$. The geometry found (Fig. 6.b.) has very close fins. In the middle the spacing is minimum and increases slightly for the outer fins. This configuration reduces the thermal resistance by $20 \%$ compared to the reference. However, this solution is unrealistic as it almost triples the pressure drop of the design. As a result the aeraulic power needed to cool the system will also triple. This shows the necessity to include the pressure drop inside the optimization process to find realistic configurations.

The case where pressure drop is minimized $(\alpha=0)$ has an opposite geometry (Fig 6.c). The spacing between the fins is constant and equal to the maximum values possible $(3.5 \mathrm{~mm})$. This result seems to be consistent as a larger spacing will decrease the average velocity between the fins. This design decreases the pressure drop by $35 \%$ and only increases the thermal resistance by $8 \%$. This design can be very interesting if the main concern of the designer is to reduce the costs of the ventilation system with only a slight impact on the temperature levels.

Between the two extreme cases, the other cases can be regrouped in two categories. The ones improving only the pressure loss $(0<\alpha \leq 0.83)$ and the ones improving only the thermal resistance $(0.86 \leq \alpha<1)$. For the first group, the optimums are close to the 
Table 1 Optimization Results (lengths in $\mathbf{m m}$ )

\begin{tabular}{|c|c|c|c|c|c|c|c|c|}
\hline $\boldsymbol{\alpha}$ & $\boldsymbol{x}_{\mathbf{1}}$ & $\boldsymbol{x}_{\mathbf{2}}$ & $\boldsymbol{x}_{\mathbf{3}}$ & $\boldsymbol{x}_{\mathbf{4}}$ & $\boldsymbol{x}_{\mathbf{5}}$ & $\boldsymbol{f}$ & $\frac{\boldsymbol{R}}{\boldsymbol{R}_{\boldsymbol{r e f}}}$ & $\frac{\boldsymbol{D P}}{\boldsymbol{D} \boldsymbol{P}_{\text {ref }}}$ \\
\hline $\mathbf{0}$ & 3.5 & 3.5 & 3.5 & 3.5 & 3.5 & 0.65 & 1.08 & 0.65 \\
\hline $\mathbf{0 . 5}$ & 3.5 & 3.5 & 3.5 & 3.1 & 2.9 & 0.86 & 1.06 & 0.66 \\
\hline $\mathbf{0 . 7 5}$ & 3.5 & 2.4 & 2.2 & 3.4 & 3.0 & 0.95 & 1.03 & 0.71 \\
\hline $\mathbf{0 . 8 3}$ & 2.7 & 3.0 & 2.3 & 1.6 & 3.1 & 0.97 & 1.01 & 0.80 \\
\hline $\mathbf{0 . 8 6}$ & 1.0 & 1.8 & 1.8 & 1.8 & 2.5 & 0.98 & 0.93 & 1.25 \\
\hline $\mathbf{0 . 9}$ & 1.3 & 1.1 & 1.7 & 1.9 & 1.9 & 0.96 & 0.90 & 1.43 \\
\hline $\mathbf{1}$ & 0.5 & 0.6 & 0.7 & 1.0 & 1.0 & 0.80 & 0.80 & 3.88 \\
\hline
\end{tabular}

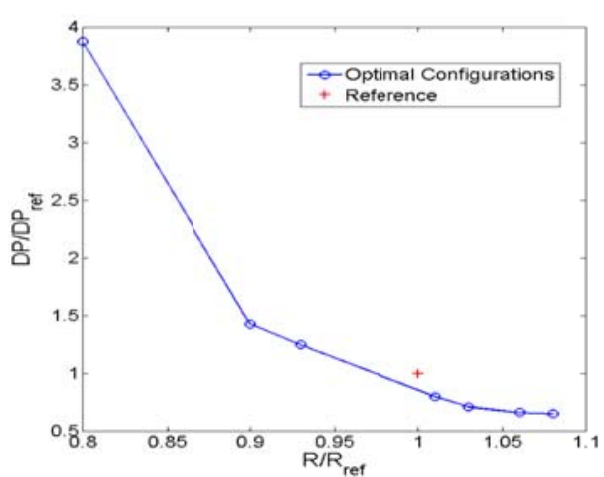

Fig. 4. Pareto front of the different optimal solutions.

case where DP is minimized. The geometries have large spacings that decrease as $\alpha$ increases. The first spacing is always maximal except for the case with $\alpha=0.83$. For all these geometries, the thermal resistance increases slightly from $1 \%$ to $6 \%$ and the pressure drop decreases significantly from $20 \%$ to $34 \%$.

The cases where the thermal resistance is reduced shares similar topologies $(0.86 \leq \alpha<1)$. The spacings are small in the middle and increase further from the center. The two geometries seem to be interesting.

They improve the thermal resistance by $7 \%$ and $10 \%$ and increase the pressure drop by $25 \%$ and $43 \%$.

In these results, no optimum which both improves thermal resistance and pressure drop has been found. This can be explained by the fact that our reference geometry is close to the optimal solutions (Fig. 5). There is not much room for improvement. The optimums improving both objectives will probably be found for values of $\alpha$ between 0.83 and 0.86

\subsection{Performances of the optimization method}

A statistical analysis of the results have been performed to measure the performances of the optimization technique. This analysis has been done only on four values of $\alpha$ as we did not have enough sample for the other values. The results can be
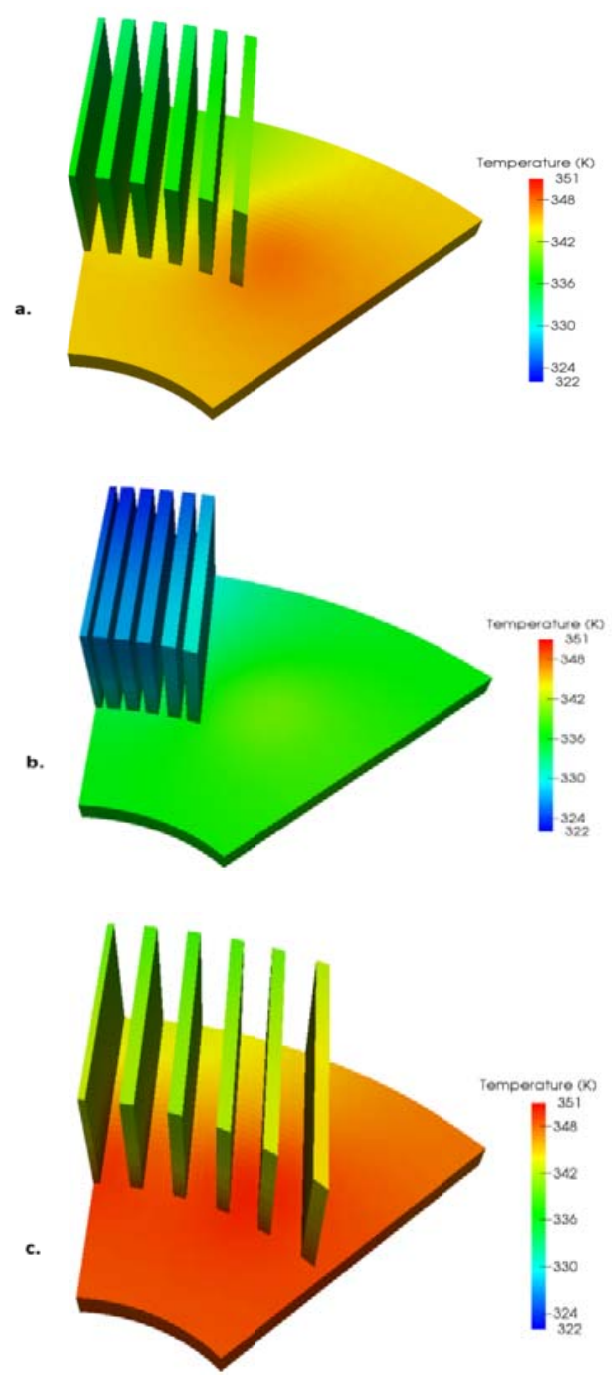

Fig. 5. ntours of temperature for the reference geometry (a). the R-optimized geometry (b) and the DP-optimized geometry (c).

found in table 2. The first indicator is the average number of function evaluation, each evaluation corresponding to a CFD calculation. Overall the average number of evaluation to find an optimum is around 475 . However, this number seems to change depending on the nature of the objective. For the case $\alpha=0$, the optimization seems faster than the cases where thermal resistance is optimized. This 
difference is also present regarding the repeatability of the method. For $\alpha=0$, the optimum found is exactly the same between all the runs. When the thermal resistance is introduced in the objective, the results start to be more scattered. The case where the results are the more scattered is when $\alpha=1$, the maximum relative difference being $5.5 \%$.

Table 2 Performances of the optimization method for four values of $\alpha$

\begin{tabular}{|c|c|c|c|}
\hline $\boldsymbol{\alpha}$ & $\boldsymbol{f}_{\text {count }_{\text {mean }}}$ & $\boldsymbol{d}_{\boldsymbol{\alpha}_{\text {mean }}}$ & $\boldsymbol{d}_{\boldsymbol{\alpha}_{\text {max }}}$ \\
\hline $\mathbf{0}$ & 374 & $0.0 \%$ & $0.0 \%$ \\
\hline $\mathbf{0 . 5}$ & 435 & $1.0 \%$ & $2.1 \%$ \\
\hline $\mathbf{0 . 7 5}$ & 570 & $0.5 \%$ & $0.8 \%$ \\
\hline $\mathbf{1}$ & 512 & $2.8 \%$ & $5.5 \%$ \\
\hline
\end{tabular}

These results can be explained by the fact that the pressure drop function has a clear minimum in our optimization space which is located directly on a boundary. On the contrary, thermal resistance seems to be very flat around the optimum. It causes the method to not converge properly. Instead of an optimal solution there is an optimal zone where the geometries are very close.

\section{CONCLUSIONS}

In the present study, a multi-objective methodology had been used to optimize a heat sink directly modelled by a three-dimensional CFD model. The method used for optimization was Variable Neighbourhood Search. The main objectives were to reduce the pressure drop and the thermal resistance of the system.

A Pareto front of the optimal configurations has been obtained. The results showed that pressure drop could be increased up to $35 \%$ and thermal resistance reduced by $20 \%$. However not including the pressure loss in the objectives gave unrealistic solution as it tripled it. We did not find any optimum improving both the thermal resistance and the pressure drop.

The optimization methods showed good performance. Overall, the average number of CFD calculations was less than 500 for one optimization run. By comparison, on a problem with similar size (same number of parameters and same equations solved by CFD), Smolka (2013) obtained results after 1200 evaluations with genetic algorithm. This seems to show that VNS gives faster results than Genetic algorithms. Moreover, the precision of the method is good: the worst optimum found had a relative difference to the best element of $5 \%$. Overall, between all the runs, the average value of this relative difference is less than $2 \%$.In the future, this methodology will be used to design more complex and irregular heat sinks which needs more computational resources to model.

\section{ACKNOWLEDGEMENTS}

The authors are grateful for the financial support provided by Valeo Powertrain Systems.

\section{REFERENCES}

Bornschlegell, A. S., J. Pelle. and S. Harmand (2013).A Fasquelle,and J.-PCorriou., Thermal Optimization of a High-Power Salient-Pole Electrical Machine, Industrial Electronics, IEEE Transactions on , 60(5), 1734-1746.

Gosselin, L., Tye-Gingras M., F. and Mathieu-Potvin.(2009). Review of utilization of genetic algorithms in heat transfer problems, International Journal of Heat and Mass Transfer 52 (9-10), 2169-2188.

Jandaud, P. O., S. Harmand., and M. Fakes(2012). Thermal design of an electric motor using Particle Swarm Optimization, Journal of Physics: Conference Series. 395, 1.

Karathanassis, I. K., E. Papanicolaou.,V. Belessiotis. and G.C Bergeles. (2013). Multi-objective design optimization of a micro heat sink for Concentrating Photovoltaic/Thermal (CPVT) systems using a genetic algorithm, Applied Thermal Engineering, 59, 733-744.

Kennedy, J., and R. Eberhart. (1995). Particle swarm optimization, Neural Networks, Proceedings of IEEE International Conference on Neural Networks, IV, 1942-1948.

Kim, T., D. Song.,S Kato., and S. Murakami (2007).Two-step optimal design method using genetic algorithms and CFD-coupled simulation for indoor thermal environments, Applied Thermal Engineering, 27, (1). 3-11.

Liberti L., and M. Drazic (2005). Variable Neighbourhood Search for the Global Optimization of Constrained NLPs, Proceedings of GO 2005, 1-5.

Lin. L., Y. Y. Chen., X.X Zhang., and X.D Wang (2014).Optimization of geometry and flow rate distribution for double-layer microchannel heat sink, International Journal of Thermal Sciences 78(0), 158-168.

Mladenovic N. and P. Hansen (1997). Variable neighbourhood search, Computers and Operations Research 24 (11), 1097-1100.

Mladenovic N. and P. Hansen (2010). Variable neighbourhood search: methods and applications,Annals of Operations Research, 175(1), 367-407.

Smolka J. (2013). Genetic algorithm shape optimisation of a natural air circulation heating oven based on an experimentally validated 3-D 
P. Jandaud et al. /JAFM, Vol. 9, Special Issue 1, pp. 31-37, 2016.

CFD model, International Journal of Thermal Sciences 71, 128-139.

Vasudev K. L., R. Sharma, and S. K. Bhattacharyya.
(2014). A multi-objective optimization design framework integrated with CFD for the design of AUVs, Methods in Oceanography, 10, 138-165. 